\title{
Diabetes and Road Traffic
}

Authors

Oliver Ebert ${ }^{1}$, Barbara Bohn², 3, Bernd Bertram4, Barbara Buchberger ${ }^{5}$, Hermann Finck ${ }^{\dagger}$, Jürgen Hoß ${ }^{6}$, Peter Hübner ${ }^{7}$, Laura Krabbe $^{8}$, Bernhard Kulzer ${ }^{9}$, Eva Küstner ${ }^{10}$, Bernhard Lachenmayr ${ }^{11}$, Klaus-Dieter Lemmen ${ }^{12}$, Friedhelm Petry ${ }^{13}$, Kurt Rinnert ${ }^{14}$, Markus Salomon ${ }^{15}$, Wolfgang Schütt ${ }^{16}$, Reinhard W. Holl2

\section{Affiliations}

1 REK Attorneys at Law, Stuttgart, Balingen, Germany

2 Institute for Epidemiology and Medical Biometry, ZIBMT, University of Ulm, Germany

3 NAKO e.V., Heidelberg, Germany

4 Ophthalmological Practice Prof. Bertram \& Dr. Helg, Aachen, Germany

5 Department of Health Technology Assessment and Systematic Reviews, University of Duisburg-Essen, Germany

6 Specialist Practice Dr. Rainer Möllmann and Dr. Jürgen Hoß, Krefeld, Germany

7 Dr. Peter Hübner, Bonn, Germany

8 Chair of Medical Management, Faculty of Economics, University of Duisburg-Essen, Campus Essen, Germany

9 Research Institute of the Diabetes Academy Bad Mergentheim (FIDAM GmbH), Bad Mergentheim, Germany

10 Gau-Bischofsheim, Germany

11 Eye Practice Clinic Prof. Dr. Dr. Bernhard Lachenmayr \& PD Dr. Lukas Reznicek, Munich, Germany
12 Ophthalmological Practice PD Dr. Lemmen \& Drs. Vahdat, Düsseldorf, Germany

13 Internist Group Practice, Wetzlar, Germany

14 Company Medical Service, City of Cologne, Germany

15 Diabetes Specialist Practice and Center for Nutritional Medicine, Medicum Hamburg, Germany

16 Diabetes Center, Imland Klinik Eckernförde, Germany

\section{Bibliography}

DOI https://doi.org/10.1055/a-1018-9262

Exp Clin Endocrinol Diabetes 2019; 127 (Suppl 1): S114-S123

(c) J. A. Barth Verlag in Georg Thieme Verlag KG Stuttgart .

New York

ISSN 0947-7349

\section{Correspondence}

Lawyer Oliver Ebert

Chairman of the Social Affairs Committee of DDG

Nägelestraße $6 a$

70597 Stuttgart

Germany

ebert_ddg@rek.de
These practical recommendations highlight important contents of the S2e guideline Diabetes and Road Traffic/Diabetes und Straßenverkehr, $1^{\text {st }}$ edition 2017 - AWMF register no. 057-026, printed in Diabetologie und Stoffwechsel 2018; 13(01): 54-97, DOI: 10.1055/ s-0043-124953. The guideline is available online at ://www. deutsche-diabetes-gesellschaft.de/filead- min/Redakteur/Leitlinien/Evidenzbasierte_Leitlinien/2017/Leitli nie_S2e_Diabetes_und_ Straßenverkehr_Endfassung.pdf. The long version of the guideline is binding and includes all appendices and literature references as well as the methodology report of the standardised literature search.

The guideline was published by the German Diabetes Society/ Deutsche Diabetes Gesellschaft (DDG) with the participation of: Social Affairs Committee of the German Diabetes Society/Ausschuss Soziales der Deutschen Diabetes Gesellschaft, Diabetes and the Eye Working Group/AG Diabetes und Auge, German Society for General and Family Medicine/Deutsche Gesellschaft für Allgemein- medizin und Familienmedizin (DEGAM), German Society for Occupational and Environmental Medicine/Deutsche Gesellschaft für Arbeitsmedizin und Umweltmedizin (DGAUM), German Ophthalmological Society/ Deutsche Ophthalmologische Gesellschaft (DOG), German Society for Psychology/Deutsche Gesellschaft für Psychologie (DGP), German Society for Transport Medicine/ Deutsche Gesellschaft für Verkehrsmedizin (DGVM), German Society for Traffic Psychology/ Deutsche Gesellschaft für Verkehrspsychologie (DGVP), Association of Diabetes Consultancy and Professional Training in Germany/Verband der Diabetes-Beratungs- und Schulungsberufe in Deutschland e. V. (VDBD), German Diabetic Association/Deutscher Diabetiker Bund (DDB), German Diabetes Federation/ Deutsche Diabetes Föderation (DDF) and German Diabetes Aid - People with Diabetes/Deutsche Diabetes Hilfe - Menschen mit Diabetes (DDH-M). 


\section{Legal Norms and Definitions}

Both private and professional mobility are important prerequisites for the participation and inclusion of people with chronic diseases in daily life. Participation in road traffic in Germany is regulated by the Driving Licence Ordinance/Fahrerlaubnisverordnung (FeV) [1]. Other relevant standards are the Road Traffic Act/StraßenverkehrsGesetz (StVG), the Road Traffic Regulations/Straßenverkehrsordnung (StVO), the Road Traffic Licensing Regulations/Straßenverkehrszulassungs-Ordnung (StVZO) and the Criminal Code/Strafgesetzbuch (StGB).

Category 1 vehicles: (A, A1, AM, B, BE, L, T) e. g. mopeds, motorcycles, light motorcycles, motor vehicles, agricultural and forestry tractors. These include vehicles up to $3.5 \mathrm{t}$ and motorcycles ("private drivers").

Category 2 vehicles: (C, C1, C1E, D, DE, D1E, FzF) C to D1E are e. $g$. lorries and buses ('professional drivers'). These are vehicles over 3.5t. FzF is the permission to carry passengers.

Driving safety refers to the situational and time-related ability to drive a vehicle. It can be quickly changed by external factors and by impairments to the driver. The terms "driving safety" and "fitness to drive" are used synonymously. Driving aptitude is understood as the ability to drive a motor vehicle in road traffic (in the sense of disposition), which is largely stable over time and independent of current situation and state parameters. This ability includes personality traits as well as psychophysical performance functions. Physical and mental defects which may impair driving safety from the outset must be taken into account when determining fitness to drive. In this context, the influence of the diabetes mellitus on driving safety and driving aptitude is also discussed, as it can be accompanied by complications and side effects caused by both disease and therapy. However, most people with diabetes meet the requirements for driving Category 1 (private drivers) and Category 2 (professional drivers) vehicles.

In addition to metabolic disorders (partly as a consequence of therapy), diabetes-related secondary diseases, diabetes-associated concomitant diseases and age-specific peculiarities can cause situations which both health and traffic at risk. Insufficient competence in dealing with illness is another risk factor.

\section{Accident Frequency in People with Diabetes}

Authors of a meta-analysis from 2016 came to the conclusion that the accident risk for people with diabetes is slightly increased in all cases. According to a review by Daniel Cox and colleagues from the USA, for which various results from reviews and meta- analyses from the years 2002-2012 were summarised, the accident risk of people with diabetes is only $12-19 \%$ higher than in the general population. This increased risk is very low compared to the effects of other diseases on road traffic accident frequency: for people with sleep apnoea syndrome, the risk of a road accident is increased by $140 \%$, and for attention deficit/hyperactivity disorder (ADHD) by as much as $340 \%$. Nevertheless, public perception seems to associate diabetes with an above-average risk potential. This is not least due to the strong media presence and the not-always-correct representation in media of diabetes and driving.
Moreover, accident data from North America are not categorically transferable to European conditions. Analyses show that diabetes per se is not associated with an increased risk of accidents, but that there are high risk groups within the affected population. People with a strong tendency to hypoglycaemia have a particularly increased risk of accidents. However, strict government regulations do not necessarily lead to an improvement of risk or danger. Studies from Denmark and the Czech Republic, for example, have shown that the introduction of the new European legislation on driving licences in 2012 has led to a reduction in reported hypoglycaemia, which could result in even worse road safety.

\section{Risk assessment and compensation options}

The majority of people with diabetes are well-suited to drive motor vehicles. However, the conditions for safe driving of motor vehicles may be restricted or waived temporarily or permanently if hazardous-to-traffic health problems exist or are to be expected due to inadequate treatment, side effects of the treatment or complications of the disease. These drivers require an individual evaluation of their fitness to drive a motor vehicle, with the question of whether their abilities meet the minimum requirements for driving motor vehicles.

When evaluating the driving safety/suitability of persons with diabetes, it is always necessary to consider the individual. A blanket evaluation based on the diagnosis of diabetes is not permissible, since the functional effects of the disease are very different. They depend, among other things, on the individual course of the disease, the complications that have already occurred, the ability to perceive the symptoms of hypoglycaemia, the treatment performed, the interaction with other diseases and the other individual conditions of the person with diabetes.

Consequently, the evaluation of fitness to drive should not only consider health restrictions but should also use a resource-oriented approach to include possibilities for compensation.

Possibilities for compensation can be justified on an individual basis, e. g. many years of experience as a driver, responsible and reflective handling of the illness, being proactive with regards to including possible risks and targeted coping strategies. Continuous glucose monitoring or conversion of a vehicle can also enable participation in road traffic.

A restriction of driving aptitude is equated with disadvantages in many social areas.

\section{Possible Adverse Effects on Driving Safety for People with Diabetes}

\section{Hypoglycaemias}

Various physical and mental conditions are necessary for driving a motor vehicle, and these can be impaired by hypoglycaemia. Depending on the degree of severity, hypoglycaemia leads to a restriction of cognitive functions of attention, perception, information processing, reaction speed, memory performance, vision and spatial perception as well as executive functions that control the planning, preparation and control of actions. The speed of information processing and the execution of complex tasks are especially impaired in hypoglycaemia. 
In severe hypoglycaemia, a massive impairment of consciousness, or even unconsciousness, can lead to a state of absolute inability to drive. This means that for people with diabetes, who are susceptible to hypoglycaemic events due to their form of therapy (insulinotropic oral antidiabetics, insulin), hypoglycaemia is an important factor which can impair driving safety. Frequent mild and severe hypoglycaemia are also a risk factor for hypoglycaemia perception disorders.

Causes of hypoglycaemia in road traffic can be:

- Error in insulin therapy (e.g. incorrect insulin dosage, dosage without prior glucose measurement, mix-up of insulin type),

- Incorrect dosage of medication (e. g. too high a dosage of medication that increases insulin secretion or insulin activity),

- Insufficient intake of carbohydrates (e. g. omitting meals with carbohydrates, errors in estimating carbohydrate content),

- Physical activity, sports (e. g. lack of compensation for glucose consumption through additional intake of carbohydrates; underestimation of after-effects of physical activity),

- Consequences of alcohol consumption or very strong psychotropic substances (e. g. alcohol consumption without considering the medium-term risk of hypoglycaemia),

- Lack of or impaired hypoglycaemic perception,

- Impaired drug clearance (e. g. impaired kidney function, impaired liver function, hypothyroidism),

- Interaction with other medicines (e. g. sulphonamides, fluoroquinolones),

- Impaired glucose counterregulation (e.g. pituitary insufficiency, Addison's disease, growth hormone deficiency),

- Reduced endogenous/hepatic glucose production (e.g. liver or kidney diseases, alcohol consumption),

- Impaired glucose absorption (e.g. gluten intolerance/coeliac, malabsorption in gastroparesis, anorexia nervosa or after surgical interventions on the digestive tract).

People with diabetes are only at risk of hypoglycaemia if they carry out their diabetes therapy with insulinotropic drugs (sulfonylureas, glinides) or insulin. Drugs of the classes biguanide (metformin), alpha-glucosidase inhibitors (acarbose), thiazolidinediones (glitazone), DPP-4 inhibitors (dipeptidylpeptidase-4 inhibitors; gliptins), SGLT-2 inhibitors (sodium-dependent-glucose-transporter-2 inhibitors) and incretin mimetic drugs (GLP-1 receptor agonists) do not generally present a hypoglycaemic risk when used as monotherapy or as combination therapy between these preparations.

The occurrence of severe hypoglycaemia is the most important single factor for accidents in people with diabetes. Published data on the frequency of hypoglycaemia and traffic accidents depending on the type of diabetes and form of therapy are presented in the long version of the guideline.

\section{Risk factor: Alcohol}

A 2004 study indicates that both hypoglycaemia and alcohol consumption can lead to cognitive impairment independently of each other. The authors found a cumulative effect in the combination of these two factors.

\section{Risk factor: Hypoglycaemia perception disorder}

An adequate perception of the first signs of hypoglycaemia is an essential prerequisite for driving safety. It enables a person to take appropriate measures (e.g. taking fast-acting carbohydrates) to stop the further drop in blood glucose and thus avoid hypoglycaemia during the drive.

There is no universal definition of functional or dysfunctional hypoglycaemia perception, since the spectrum exists ranging from a functioning to a completely malfunctioning hypoglycaemia perception, which, however, is reversible to a certain degree through certain measures (e. g. strict avoidance of low glucose values). Depending on the possibility of reversibility of the hypoglycaemia perception disorder, a distinction is therefore made between a "partial" and an "irreversible" disorder. A partial limitation of hypoglycaemia perception may already be present after a single hypoglycaemia.

In the presence of a hypoglycaemia perception disorder, the development of adrenergic early symptoms is reduced and the threshold of sympathetic adrenergic counterregulation lowered (hypoglycaemia-associated autonomic failure, HAAF). Often the adrenergic activation threshold falls below the neuroglycopenic threshold; this can lead to a limited ability to act behind the wheel due to the onset of neuroglycopenic symptoms and a limited ability to act and treat the hypoglycaemia. A partial hypoglycaemia perception disorder is reversible and can be treated by strictly avoiding low glucose levels so that the glycaemic thresholds of hormonal counterregulation are improved or restored (short- to medium-term increase in the $\mathrm{HbA} 1 \mathrm{c}$ value).

The risk of a hypoglycaemia perception disorder increases with the duration of diabetes or with the duration and/or intensity of insulin therapy.

\section{Evaluation of driving safety}

Steering and speed variables are usually tested for the verification of driving safety in studies with a driving simulator. In an experimental study of 25 subjects with type 1 diabetes, significantly more driving errors occurred in all steering variables at glucose levels below $50 \mathrm{mg} / \mathrm{dl}(2.8 \mathrm{mmol} / \mathrm{l})$. The participants drove slower and every third participant (35\%) displayed a deterioration in driving performance. However, only about half of those affected recognised these impairments, and one in four still considered themselves to be safe to drive and would have continued to drive in everyday life.

\section{Measures to restore and ensure driving safety}

There are a variety of measures to reduce the risk of hypoglycaemia which can also restore the driving safety of people with an increased risk potential for hypoglycaemia caused by more frequent severe hypoglycaemia or the presence of a hypoglycaemia perception disorder.

Optimization of diabetes therapy to minimize the risk of hypoglycaemia

- Systematic analysis of the causes of hypoglycaemia,

- Optimization of blood glucose/glucose measurement frequency,

- Optimization of insulin therapy,

- Optimization of glucose target values with the aim of avoiding hypoglycaemia,

- Use of drugs or insulin preparations or the choice of a therapy with a lower risk of hypoglycaemia, 
- Strict avoidance of low glucose levels and hypoglycaemia to restore the adrenergic response and perception of hypoglycaemia through increased thresholds of hypoglycaemia perception.

Use of insulin pump and continuous glucose monitoring (mainly in people with type 1 diabetes)

- Continuous glucose monitoring (CGM), in which glucose is continuously measured but the values must be actively retrieved by the patient. In addition to the current BG value, a trend arrow on the reader warns of a drop in the glucose concentration in blood.

- Real-time-continuous glucose monitoring (rtCGM), in which an alarm is issued when the glucose falls below defined threshold values, thus providing warning of a pending hypoglycaemia.

- Insulin pumps (CSII),

- Use of insulin pump and CGM/rtCGM (SaP = sensor-augmented pump therapy),

- Insulin pumps with integrated continuous glucose monitoring that issue an alarm in the case of a hypoglycaemia risk or if the glucose level falls below defined threshold values and thus warn of hypoglycaemia (CSII + rtCGM),

- Insulin pumps with integrated continuous glucose monitoring, which automatically interrupt the insulin infusion for a certain period of time when the blood glucose level drops sharply, thus minimising the risk of hypoglycaemia (LGS or PLGM).

Structured diabetes training

- Acquiring skills to better manage diabetes,

- Minimization of treatment errors leading to hypoglycaemia,

- Conveying of knowledge and skills for prophylaxis and early detection of hypoglycaemia,

- Better knowledge and abilities to reduce the risk of hypoglycaemia,

- Establishment of effective strategies for the prevention of hypoglycaemia when driving motor vehicles, participating in road traffic as well as behavioural strategies for the occurrence of hypoglycaemia while driving etc.,

- Inclusion of relatives, contact persons,

Hypoglycaemia perception training

- Conveying a plausible explanatory model for the development and treatment of problems of hypoglycaemia perception,

- Training for better perception of first signs of hypoglycaemia,

- Conveying knowledge and skills for better prevention of hypoglycaemia and for dealing with hypoglycaemia,

- Development of individual strategies to avoid hypoglycaemia

- Inclusion of relatives, contact persons,

Behaviour for the prevention of hypoglycaemia

- Check blood glucose before and during the drive. The glucose level should not be less than $90 \mathrm{mg} / \mathrm{dl}(5 \mathrm{mmol} / \mathrm{l})$ before driving. If the blood glucose level is below this value, the blood glucose level should be raised by ingesting carbon dioxide. For longer drives, blood glucose should be measured at minimum every $3 \mathrm{~h}$. More frequent measurements are necessary due to the lower limit values, e. g. for women with gestational diabetes on insulin.

- Taking along foods which contain carbohydrates (“Hypo BE/ CC").

- Documentation of blood glucose values and treatment measures,

- If there are signs of hypoglycaemia or a measured blood glucose level of $<70 \mathrm{mg} / \mathrm{dl}(<3.9 \mathrm{mmol} / \mathrm{l})$, the driver must be assumed to be unfit to drive, so that the journey should be interrupted immediately in order to treat the hypoglycaemia.

For the acute treatment of hypoglycaemia, at least 2 CC/BE fastacting carbohydrates (e.g. dextrose, fruit juice/soda) should be ingested. After this immediate measure, blood glucose should be checked; if the test confirms hypoglycaemia, it is recommended to ingest a slow-acting $\mathrm{KE} / \mathrm{CC}$ to prevent a renewed drop in glucose.

The journey must not be restarted until cognitive performance has been completely restored (at the earliest after $20 \mathrm{~min}$ ).

After the treatment of hypoglycaemia, regular blood glucose checks should be performed until it is sure that the blood glucose level is in fact above $90 \mathrm{mg} / \mathrm{dl}$ and stable.

In the context of medical care for people with diabetes who participate in road traffic, it is necessary to explicitly pose the question on a regular basis as to the occurrence of hypoglycaemia in road traffic or the occurrence of driving/traffic anomalies or accidents.

\section{Severe acute metabolic disorders, chronic hyperglycaemia and brittle diabetes}

In contrast to hypoglycaemia, hyperglycaemic blood glucose values that remain constant have no proven significant influence on driving safety according to the current state of studies. In most controlled studies (partly with glucose clamp technique) on the influence of hyperglycaemia, no loss of cognitive performance or changes in mood were found. However, a reduced speech ability and a reduced IQ as well as mild cognitive changes in the sense of slower information processing processes and memory were observed in some persons in the state of hyperglycaemia.

Serious metabolic derailment caused by insulin deficiency, i.e. diabetic ketoacidosis, can lead to cognitive impairments (dizziness, impaired consciousness) that severely impair driving safety or make it impossible to drive a vehicle.

When long-term severely high blood glucose values are rapidly reduced (e. g. by insulin), patients' vision may be impaired by fluctuations in the refractive power of the lens due to the osmotic effects of the increased blood glucose (blurred vision). This can impair the driving safety of affected patients for up to a duration of 3 months. In cases of doubt, an individual evaluation by a medical specialist should be carried out to assess the patient's vision.

People with diabetes should be informed about the hyperglycaemia risk of their diabetes therapy and the associated possible restrictions on driving safety.

\section{Diabetic secondary diseases}

Eye diseases/eye disorders

Diabetes can lead to changes in the retina, eye muscle paresis, accommodation disorders and cataracts. Every person with diabetes 
should be regularly examined by an ophthalmologist in accordance with the national care guideline "Prevention and therapy of retinal complications in diabetes/Prävention und Therapie von Netzhautkomplikationen bei Diabetes".

Patients with advanced diabetic retinopathy or maculopathy should only drive a vehicle if they have sufficient vision according to the FeV. To evaluate driving safety in people with diabetes, a current ophthalmological examination result is to be used. In the case of advanced diabetic retinopathy, diabetic maculopathy or impaired vision from other causes, the ophthalmologist should check and determine driving safety with regard to visual function.

For people with diabetes with newly occurring symptoms such as deterioration of vision, distorted vision, blurred vision or floaters, a prompt examination by an ophthalmologist should be carried out. Patients should be made aware that a visual impairment means they could be unsafe to drive.

If people with diabetes become insecure drivers as a result of diabetic maculopathy and there is an indication for intravitreal therapy with VEGF inhibitors, treatment can restore driving safety and the necessary visual acuity sooner than without therapy. Treatment with extensive laser coagulation, also as part of a vitrectomy, can lead to restrictions in the field of vision, which can reduce or exclude the ability to driving. It must be noted that the restriction of the visual field depends on the number, position and size of the grid laser photocoagulation. The basis for this therapy indication in advanced retinopathy is that retinal areas are not perfused, so that even without laser treatment, visual field reductions can often occur. In rare cases, diabetes can also cause external muscle paresis with the consequence of double-vision (diplopia). These are often reversible within weeks to months, but can lead to a loss of driving safety for that time period.

A more detailed description of the background and evidence of the recommendations can be found in the national care guideline "Prevention and therapy of retinal complications in diabetes/Prävention und Therapie von Netzhautkomplikationen bei Diabetes".

\section{Diabetic neuropathy}

Despite the absence of valid population-based surveys, a high prevalence of peripheral polyneuropathy (PNP) (8-54 \%) can be regarded as assured. People with neuropathy have an increased frequency of accidents. Peripheral polyneuropathies (PNP) can cause severe dysfunctions which compromise the safe use of pedals. After a functional anamnesis and clinical examination and in the case of clinically-relevant functional limitations, the patient should be informed that he is no longer suitable for driving a motor vehicle due to his health impairments (a so-called medical ban on driving).

Several studies have shown that patients with therapeutic immobilization of a lower extremity (e. g. total contact casting in cases of diabetic foot ulcer or neuropathic arthropathy) have severe functional deficits, especially in safe pedal use. These restrictions are so severe that a medical ban on driving is imposed for the entire period of immobilisation.

Patients with left-sided immobilisation may be allowed to use an automatic vehicle. If it is necessary to retrofit a motor vehicle in order to ensure professional participation, benefits can be claimed from the German Pension Insurance, possibly also from the Federal Employment Office or the Integration Office.
In the case of symptomatic cardiac arrhythmias within the framework of autonomous neuropathy, several cardiological societies call for a (at least temporary) "medical ban on driving".

In the case of serious other neurological complications (e. g. impaired vision due to ocular nerve palsy, dizziness in autonomous cardiac neuropathy), a medical examination should be carried out to clarify driving safety.

\section{Relevant diabetes-associated concomitant diseases}

\section{Cardiovascular diseases}

Heart disease is a common and serious secondary disease in people with diabetes. However, there is no reliable data available for Germany on accident frequencies in causative heart diseases. Data from the USA and Canada suggest that only a very small percentage of traffic accidents stem from cardiac causes. Heart diseases, especially the group of cardiac arrhythmias, can lead to serious disturbances of consciousness, which endanger both the driver and others on the road. In German-speaking countries, the German Society for Cardiology - Cardiovascular Research/ Deutsche Gesellschaft für Kardiologie - Herz- und Kreislaufforschung (DGK) published a position paper in 2010 that reflects the state of knowledge and is intended to make it easier for all physicians, evaluators of fitness to drive and patients to make decisions on fitness to drive in the event of cardiovascular diseases. The recommendations are based on the experience of experts and the interpretation of clinical studies that were not primarily performed to evaluate the driving suitability of patients. In the position paper, these experts give their opinion on driving aptitude for patients with various forms of cardiac rhythm disorders, pacemakers and ICDs, coronary heart disease including bypass surgery, acute myocardial infarction, heart failure, aortic aneurysm, cardiomyopathies and heart valve defects as well as arterial hyper- and hypotonia.

It is important, from a diabetological point of view, to understand that people with diabetes and a tendency to hypoglycaemia have an increased cardiovascular risk.

People with diabetes and cardiovascular concomitant diseases should be informed and advised about this correlation and driving aptitude. During the consultation and evaluation process, the evaluation guidelines for driving aptitude should be taken into account.

\section{Sleep apnoea syndrome}

Sleep apnoea is a very common and medically traffic-relevant comorbidity of type 2 diabetes. It is present in 23 to $58 \%$ of those affected. Validated and established instruments (Epworth Sleepiness Scale (ESS), Berlin questionnaire) are available for screening purposes. All patients with suspected sleep apnoea syndrome (SAS) or with a confirmed diagnosis should be asked about the presence of daytime sleepiness, especially about a tendency to fall asleep during everyday activities and about past accidents or near accidents associated with a tendency to fall asleep.

The presence of sleep apnoea increases the accident risk by a factor of 1.2-4.9. Sleep apnoea is one of the causes of up to $16 \%$ of road traffic accidents. Patients with increased daytime sleepiness should be informed immediately about the possible risks of driving until an effective therapy is carried out.

The evaluation guidelines for driving aptitude describe the diagnostic procedure for suspected sleep apnoea syndrome and the 
criteria for driving aptitude. If left untreated, the driver's driving aptitude is compromised in the event of noticeable daytime sleepiness. A value of at least 11 out of 24 points in the ESS is assumed to be conspicuous daytime sleepiness. After successful therapy (usually with a CPAP (Continuous Positive Airway Pressure) device) and expert documentation, driving aptitude may be fully or partially restored under certain conditions.

\section{Depression}

Depressive illnesses occur more frequently in people with diabetes than in metabolically healthy people. There are restrictions on driving safety in depression, especially in severe depressive disorders, manic episodes and bipolar affective disorders. According to the guiding principles of the evaluation guidelines on motor fitness/ Begutachtungsleitlinie zur Kraftfahreignung BASt (Section 3.12.4 Affective Psychoses), Category 1 drivers are generally expected to behave appropriately when participating in road traffic with a motor vehicle after the manic phase has subsided. In the case of several manic or very severe depressive phases within a short interval, it is not to be assumed that the road traffic behaviour when driving a motor vehicle will be appropriate, since the further course cannot be foreseen (even if there are no disturbances at present). Appropriate driving behaviour can only be reassumed if the disease activity has decreased and a progression to the degree of severity is no longer expected. This shall be evidenced by regular psychological checks. Category 2 drivers must be free of symptoms. As already formulated for drivers in Category 1 , after several depressive or manic phases, it is generally not to be assumed that they will behave appropriately when participating in road traffic with a motor vehicle.

Treatment with antidepressants can lead to restrictions in driving safety; on the other hand, taking the medication enables successful treatment of depression. Investigations into the driving safety of depressive patients show that newer, selective antidepressants have a more favourable influence on psychomotor performance parameters than tricyclic and tetracyclic antidepressants, which have a sedative effect.

Selective serotonin reuptake inhibitors (SSRIs) are also recommended for the treatment of depression in diabetes because of their more favourable side-effect profile and should also be used as a matter of priority for people with diabetes in view of the lower impairment of driving safety. Due to the sedative effect of tricyclic and tetracyclic antidepressants, they should only be prescribed with regard to driving safety if there is a specific indication (e. g. diabetic neuropathy) or if the patient wishes to use tricyclic drugs.

The doctor prescribing antidepressants is obliged to inform the patient of any side effects that may impair driving safety. The evaluation of possible restrictions of driving safety in depressions should take into account the clinical picture and course of the disease, the individual reaction to the prescribed antidepressant and the dosage.

\section{Dementia}

Dementia as a result of a mostly chronic or progressive disease of the brain with the disruption of many higher cortical functions (including memory, thinking, orientation, conception, arithmetic, ability to learn, language, speaking and decision-making) occurs more frequently in people with type 2 diabetes than in the comparison population. Type 2 diabetes - often associated with hypertension, hyperlipidaemia and obesity - is a risk factor for the occurrence of dementia. The cognitive disorders affect driving safety and driving aptitude. Restrictions in driving safety result from the specific symptoms of dementia: limited ability to react, attention and orientation disorders, reduced ability to grasp complex situations quickly and impairments as a function of the cerebral lesion pattern (e. g. disorders of spatial vision, motor coordination). In advanced stages of the disease, the consequences of cognitive impairments in terms of driving safety can no longer be realistically assessed by the patient, and there is an increased risk of traffic accidents. Furthermore, a cross-sectional study showed that the rate of hypoglycaemia is significantly higher in people with type 2 diabetes and dementia compared to people with type 2 diabetes without dementia.

Dementia at an early stage is not necessarily associated with a loss of driving safety or driving aptitude, and there is no defined limit in the light to moderate dementia where driving aptitude ends. Due to the individual variability of functional losses in dementia as well as the compensation strategies, there is also no gold standard for the clarification and evaluation of driving safety/aptitude in dementia. The stage of severe dementia is no longer compatible with driving a motor vehicle. When evaluating individual driving safety/aptitude, a detailed anamnesis of the person concerned and an external anamnesis of relatives or contact persons are necessary. The results of neuropsychological tests cannot alone justify the decision on driving safety/aptitude. The evaluation of driving safety/aptitude should be repeated at regular intervals due to the progressive course of dementia.

\section{Age-specific characteristics}

Adolescents and young adolescents

In Germany at the age of 15 , it is possible to acquire a driving licence for a moped and participate in motorised road traffic. In 2015 , a total of 19820 young people aged $15-17$ were involved in accidents in Germany. The main cause is considered the loss of control over the two-wheeler due to, among other things, not adjusting the speed or misjudging the course of the road. An analysis by the Federal Statistical Office/Statistischen Bundesamt in 2015 showed that the accident risk was highest among all drivers in the 18-24 age group; $16.7 \%$ of all injured and $13.7 \%$ of all fatally injured in road traffic belonged to this age group. The main reasons given for the high accident risk, in addition to less experience at the wheel, are the overestimation of personal abilities. One additional factor in people with diabetes is that in adolescence and young adulthood, metabolic control is less favourable than in later adulthood: the HbA1c values are higher and more metabolic derailments occur. Furthermore, diabetes self-management and therapy adherence in adolescents and young adults are often lower.

For adolescents with metabolic disorders who wish to have a driving licence, metabolic control should first be optimised, if necessary, by changing the treatment regime. Doctors and other medical specialists should be sure to inform their patients and their parents about possible effects of diabetes on driving safety in early adolescence ( 14 years and older). The risk should be individually assessed and documented together with those affected. 
The student driver should inform the instructor about his illness and its possible effects on driving safety. Leaflets can be helpful here. The student driver should inform the accompanying person about necessary assistance measures in the event of (severe) hypoglycaemia.

In the case of accompanied driving from the age of 17, the new driver should inform the accompanying person about his illness and its possible effects on driving safety. Leaflets can be helpful here. The new driver should inform the accompanying person about necessary assistance measures in the event of (severe) hypoglycaemia. Before starting the drive, the accompanying person should have clarified at least the following questions with the driver:

- Is the blood glucose in the unproblematic range?

- Is there a BG measuring device in the vehicle?

- Are fast-acting carbohydrates available in the vehicle and easily accessible?

The accompanying person should, just as the new driver, be able to recognise early symptoms of hypoglycaemia and support the new driver in the onset of hypoglycaemia with appropriate measures.

\section{People with diabetes in advanced adulthood}

Another group with a proven increased risk of accidents among the general population is the elderly. The report of the Federal Statistical Office describes that

"Older people [...] lose the overview in complex situations more quickly than road users in younger age groups";

the main causes of accidents - right-of-way errors, mistakes when turning, turning around, reversing, entering traffic and starting after coming to a halt- indicate age-related impairments of perception rather than reckless behaviour. In elderly people with diabetes, this is aggravated by the fact that, in addition to more frequent diabetes-associated secondary diseases and comorbidities, hypoglycaemia perception appears to continuously decrease. This is partly compensated by a more defensive driving style and greater driving experience.

People with diabetes at an advanced age who are treated with hypoglycaemia-inducing substances (especially insulin, sulfonylurea) should pay particular attention to the occurrence of hypoglycaemia while driving. This should be repeatedly addressed to the elderly patients by the treating medical staff/ consultants and documented in the medical record. Older people with diabetes who participate in road traffic and who are suspected of having reduced hypoglycaemia perception should be advised not to drive, to participate in hypoglycaemia perception training or take other measures.

\section{Requirements for Patient Information}

\section{Patient training}

Road safety requires the acquisition of specific knowledge, skills and codes of conduct which maintain driving aptitude in the long term, reduce the risk of critical events occurring while driving and enable the competent evaluation of one's own driving safety.
Structured diabetes education has been shown to have positive effects on the reduction of hypoglycaemia and on diabetes-related knowledge and skills. Therefore, the national guideline for structured training in diabetes calls for every person with diabetes to be trained as soon as possible after the manifestation of the disease, when changing over to a new therapy regime and in the event of problems or special challenges in diabetes therapy. Depending on age and therapy concept, participants require different contents of traffic medical consultation and information. The participants' previous experience with hypoglycaemia and changes in the probability of occurrence through therapy optimisation must be taken into account. In particular, patients who have not yet experienced hypoglycaemia should be informed about a higher risk resulting from a change in therapy and a reduction in blood glucose levels. They must be guided to compensation options such as more frequent blood glucose self-monitoring, especially before driving, and necessary carbohydrate intake at a blood glucose level below the target value.

Not every training programme explicitly deals with the aspects of safe participation in road traffic (see Practice tool 1 in the Appendix). In this case, the training staff and patients must transfer the contents of the training programme to the behaviour in road traffic.

The Blood Glucose Awareness Training (BGAT) is a psychoeducative group training; it has been available since 1997 as a German version of the original BGAT by Cox et al. One programme developed and evaluated in Germany is the "HyPos" Hypoglykämie Positives Selbstmanagement programme (hypoglycaemia - positive self-management).

People with diabetes should be informed that they should not drive during a metabolic derailment that is associated with severe restrictions in their reactions, sensory perception or general condition. People with diabetes should be informed that they should not drive a vehicle if there is a temporary reduction in driving safety during an initial phase of adjustment to antidiabetic therapy or a change in diabetes therapy as a result of blood glucose fluctuations with hypoglycaemia, accommodation disorders or other adjustment disorders. In addition, people with diabetes should be informed about how secondary or concomitant diseases can affect their driving ability.

An overview of tips on how to behave and safety rules for safe driving can be found in Practice tool 2 (see Appendix).

\section{Information by the doctor/diabetes team}

The obligation to provide comprehensive information arises initially from the treatment contract, from the Patient Rights Act/Patientenrechtegesetz ( $\S 630 c$ and $\S 630$ e BGB = Bürgerliches Gesetzbuch; engl. GCC = German Civil Cod) and from professional regulations (including $\S 8 \mathrm{MBO}$ MBO- $\ddot{A}=$ Musterberufsordnung der $\ddot{\text { Arzte }}$ in der Fassung MBO-Ä 1997, in der Fassung der Beschlüsse des 121. Deutschen Ärztetages in Erfurt, zuletzt geändert durch Beschluß des Vorstandes der Bundesärztekammer am 14.12.2018, engl. (Model) Professional Code for Physicians in Germany - MBO-Ä 1997 - The Resolutions of the 121st German Medical Assembly 2018 in Erfurt as amended by a Resolution of the Executive Board of the German Medical Association on 14/12/2018), amongst others. The 
patient should be warned of disease-related risks and protected against avoidable dangers in the best possible way.

\section{Form of information}

According to $\S 630$ e BGB, the information must be provided orally by the practitioner or by a person who has the necessary training to carry out the measure; in addition, reference can also be made to documents which the patient receives in text form.

It is therefore not sufficient to simply present the patient with a written information sheet or to have him sign such a sheet. A verbal information discussion is indispensable. It is strongly recommended for evidence purposes that the patient additionally be informed and educated in writing. In particular, if a "medical ban on driving" has to be granted, this should be communicated in writing for liability reasons in order to eliminate misunderstandings or ambiguities regarding the content of this information. Finally, the doctor must also ensure that the information is understandable for the patient. If necessary, a person who is fluent in the language or an interpreter is to be called in according to the intention of the law (BT-Drs. 17/10 488, p. 25).

\section{Documentation}

The physician is obliged to provide comprehensive and orderly documentation in accordance with $\S 630 \mathrm{f}$. BGB, amongst others. For this purpose, a patient file - in paper or electronic form - must contain all measures and their results which are important from a professional point of view for the current and future treatment including, in particular, anamnesis, diagnoses, examinations, examination results, findings, therapies and their effects, interventions and their effects, consents and information. A lack of documentation categorically justifies the assumption that a treatment measure or clarification has not taken place.

The documentation should contain the main content of the information discussion, including the recommendations and evaluations made. If a medical ban on driving must be imposed due to a temporary inability to drive, the documentation should show beyond any doubt that the patient has been informed accordingly and is thus aware that he is not allowed to drive a vehicle for the time being despite having a valid driving licence. The documentation should include the place, date, time, and duration of the information discussion and, if applicable, the persons present.

For evidence purposes it is advantageous if the patient confirms the information or instruction about his inability to drive with his signature (Practice tool 3, see Appendix). However, there is no obligation for the patient to sign such a document.

Consequences of insufficient information or failure to provide information

The attending physician must reckon with claims for damages and compensation for pain and suffering if the patient for lack of information has not recognised the risks caused by the illness and therapy and he therefore erroneously assumed that he was safe to continue driving. In this context, claims for damages can be made not only by the patient himself, but in case of doubt also by regulating insurers, by third parties injured by the accident or by health insurance companies. If the failure to provide information leads to an accident resulting in death or personal injury, there may be a risk of criminal consequences, in particular pursuant to $\S \S 222,13 \mathrm{StGB}$ (involuntary manslaughter) or $\S \S 229,13$ StGB (physical injury resulting from negligence).

On the other hand, the doctor is not responsible for any breach of duty on the part of his patient.

If the patient has been effectively informed that, due to his health restrictions, he may not (or any longer) participate in motorised traffic, but disregards this and drives a motor vehicle regardless, then the doctor is not at fault and does not have to worry about liability in such a situation either. In such cases, the patient is usually liable to prosecution - even without an accident - according to $\S 315$ c StGB, because he drove a vehicle, although he was properly informed that he was not in a position to do so for health reasons. In the event of an accident, the patient is also liable to prosecution in accordance with further standards (including $§ \S 222,229$ StGB), the exclusion of benefits or recourse by liability insurers because ignoring well-founded medical instructions is generally regarded as gross negligence.

\section{Requirements for Evaluations}

\section{Traffic medical evaluations}

\section{Occasion and implementation}

According to the FeV, under certain circumstances (e. g. for certain diseases listed in Appendix 4 or 5 of $\S 11$ and disclosure of these diseases to authorities), drivers must undergo a medical evaluation (e. g. after an accident) in order to prove that they are fit or safe to drive. Refusing the examination results in the loss of the driving licence. The evaluator can be selected by the person concerned. It is only in the case of a judicial evaluation that the court is responsible for the selection.

When applying for the issue or renewal of a driver's licence for motor vehicles in driving licence Category 2, people with diabetes are always required to undergo a medical examination. In doing so, the evaluator must adhere to the questions on motor vehicle licensing by the driving licensing authority. This investigation may only be carried out in accordance with recognised scientific principles (FeV, Appendix 4a, para. 1c). The evaluation must be written in language that is generally understood, comprehensible and verifiable. All essential findings leading to the evaluation must be included and the testing procedure must be specified. The evaluation must be complete in all essential points, in particular with regard to the questions posed. A distinction must be made between previous medical history and current findings. The costs of the evaluation shall be borne by the person under examination. The evaluation is distributed to the person under examination unless the evaluator has been commissioned by a court of law.

\section{Legal basis}

In addition to the current state of scientific knowledge, the medical evaluator must above all consider the legal bases. In the field of traffic medicine these are the Criminal Code/Strafgesetzbuch $\S$ $315 c$ (endangerment of the road traffic/Gefährdung des Straßenverkehrs), the Road Traffic Act/Straßenverkehrsgesetz and the Driving Licence Ordinance/Fahrerlaubnisverordnung, in particular $\S \S$ 11 to 14 , which regulate the health suitability and the aptitude for 
driving a motor vehicle. The special aptitude for diabetes is regulated in Appendix 4.

Because they are based in the FeV, Annex 4a, the evaluation guidelines for driving aptitude of the BASt also have a normative character [2] and are therefore mandatory. An updated version, in which the chapter Diabetes mellitus was also revised (Practice tool 4, see Appendix), was published on 24 May 2018. The guidelines represent the current state of science and technology. If they are applied, no explicit justification is required. Deviations from the guidelines are possible, but usually require detailed justification.

\section{Scientific foundations}

The evaluator must take into account the current state of scientific knowledge. This is of particular importance if it deviates from the normative requirements in evaluating individual cases.

\section{Qualification of the evaluator}

The evaluation can be carried out by a

a) Specialist with qualifications in traffic medicine,

b) A doctor from the public health department or another doctor from the public administration,

c) Specialist in forensic medicine,

d) Physician with the regional designation "Occupational Medicine" or the additional designation "Works Medicine",

e) Doctor at a driving aptitude evaluation centre.

For certain questions, one or more additional qualifications (e. g. specialist in internal medicine and additional qualification in diabetology) may be required. The physician performing the evaluation should not be the attending physician of the person under examination in order to exclude bias. Addresses of diabetologists with traffic medicine qualifications can be found on the website of the German Diabetes Society/Deutsche Diabetes Gesellschaft (DDG) (https://www. deutsche-diabetes-gesellschaft.de/arztsuche).

\section{Scope of examination for diabetes}

The focus of the questions to be answered by the evaluator is the examination of the question whether the driver with diabetes is restricted in his driving aptitude/driving safety due to his disease. This requires an exact anamnesis and includes the following questions:

- Have severe hypoglycaemia (with outside help) occurred in particular in the last 12 months?

- Is there an increasing incidence of hypoglycaemia?

- Has hypoglycaemia perception decreased?

- Is a sufficient self-check including documentation being carried out?

- Have there been any training courses?

- Is disease self-management (especially in hypoglycaemia and road traffic) well-controlled?

- Are there regular medical check-ups?

\section{Verdict}

Legally, the evaluator only has the position of an advisor for the authority or court which makes the decision. In the non-judicial evaluation, the person concerned is the addressee and not the authority. The evaluation can only be passed on to the authority or third parties with the consent of the person concerned. In the preparation of such an evaluation in traffic medicine in Germany, it is important to note that, in addition to the Driving Licence Ordinance, the evaluation guidelines for driving aptitude, which have a normative character, are legally binding. The core statements specified there on diabetes are the following:

- People with diabetes who have received proper training and who display good metabolic control can safely drive vehicles of both categories.

- Therapy regimes and vehicle use must be taken into account in the evaluation.

- The main concern regarding driving safety in diabetes is based on the occurrence of hypoglycaemia with loss of control, behavioural disorders or impaired consciousness.

- An unrestricted perception of hypoglycaemia is a prerequisite for driving aptitude.

- People with diabetes with more than one daytime hypoglycaemia requiring external help over the last 12 months are generally unsuitable for driving a motor vehicle until a sufficient metabolic stability as well as a reliable perception of hypoglycaemia are regained and ensured.

- Anyone who has received adjustment after a metabolic decompensation or who has been newly adjusted may not drive a vehicle until the adjustment phase has been completed by reaching a balanced metabolic state (including normalisation of vision) [2].

In the case of diseases of several organ areas (e. g. secondary and/ or concomitant diseases of diabetes), the relevant sections of the evaluation guidelines for driving aptitude must also be taken into account.

Possible compensation for lacking suitability is expressly provided for. With regard to diabetes, measures are specified such as hypoglycaemia perception training, changes in therapy or increased blood glucose self-monitoring, which can be incorporated as conditions into an evaluation report by a medical (diabetological) specialist. New techniques such as continuous glucose monitoring (CGM/rtCGM) or the use of insulin pump systems with hypoglycaemia warning function are also possible as compensation. Hyperglycaemia only excludes the option of driving if there are pronounced symptoms such as fatigue, loss of consciousness or impaired vision.

The evaluation also depends on the therapy regime, adjustment and vehicle use. The drugs must be differentiated according to substances with a low (e. g. metformin, biguanide, incretin-based substances) or higher (e. g. sulfonylureas and their analogues) or high risk of hypoglycaemia (insulin). Vehicle use must be taken into account, e. g. in terms of driving performance, time pressure, passenger transport or the transport of dangerous goods. Since the traffic medical risk can change rapidly over the course of the diabetic disease, the evaluator is required to specify a follow-up examination period of 3 years for driving licences for Category 2 vehicles for people with diabetes who are treated with medication and who have a higher or high risk of hypoglycaemia. Regular medical checks and self-monitoring of the metabolism are to be requested, if necessary. A well-kept, plausible blood glucose journal can be submitted to demonstrate a balanced metabolic control/self-monitoring. 


\section{Company medical opinion}

In our society, adequate mobility is largely required in both the professional and private spheres (social participation). Particularly in the professional context, access to jobs can become difficult in cases of limited or lacking safe driving abilities. In particular when it becomes necessary to give up jobs involving driving, it usually leads to occupational disability and thus often to social decline.

Conditions that can influence driving aptitude/safety in people with diabetes must first be analysed within the framework of the evaluation of working conditions in accordance with $\S 5$ ArbSchG. These can be structured as follows:

Disease-specific risks, e.g:

- Danger to oneself or others resulting from severe, in particular recurrent hypoglycaemia

- Manifestation of other diseases as a result of diabetes (microand macroangiopathy, neuropathy) or an accompanying disease (e. g. sleep apnoea syndrome)

Activity-specific risks, e.g:

- Difficulty in plannability of the daily routine and metabolic self-control (e. g. driving under persistently high time pressure)

- Occupational situations that favour or exacerbate the occurrence of acute or chronic consequences of diabetes

The disease-specific risks also include diseases which, as already mentioned above, occur as a result of diabetes (e. g. retinopathy, nephropathy) or as an concomitant disease (e. g. sleep apnoea syndrome) and can lead to functional restrictions. The expected functional limitations must be closely monitored and controlled.

Driving activities in which the daily routine cannot be sufficiently planned in advance can put strain on an adequate treatment - for example through very irregular mealtimes, significantly varying physical strain or also through the difficulty of metabolic self-control. The risk of hypoglycaemia is higher in professions whose working conditions prevent the necessary intake of carbohydrates (e.g. working under great time pressure, as a first-responder, or in parcel delivery). For these professions and activities, it is particularly true that personalised patient training can alleviate some of the restrictive conditions or make them irrelevant. For driving activities that are associated with particular stress and depending on the results of the evaluation of the workplace hazards, additional protective measures for people with diabetes are generally sufficient [3]. As a result of the rapid further development of continuous glucose monitoring with glucose sensors, often in combination with the use of insulin pumps, these new techniques will become increasingly important in the future for risk minimisation in the professional context and will require a continuous re-evaluation of acceptable risks in the professional context, as relevant risks can be minimised or even excluded.

Further recommendations for the evaluation of workplace and occupational-specific risks can be found in the guidelines for Oc- cupational doctors on diabetes and profession/Leitfaden für Betriebsärzte zu Diabetes und Beruf, issued by the German Statutory Accident Insurance/Deutsche Gesetzliche Unfallversierung e. V.

\section{Legal Basis}

For driving aptitude/safety, the following standards or standardslike specifications in particular must be taken into account:

- Criminal Code §315c (Endangerment of Road Traffic)/ Strafgesetzbuch § 315c (Gefährdung des Straßenverkehrs)

- Road Traffic Act (StVG)/Straßenverkehrsgesetz (StVG)

- Driving Licence Ordinance (FeV)/Fahrerlaubnis-Verordnung (FeV), https://www.gesetze-im-internet.de/fev_2010/

- Evaluation guidelines for driving aptitude/Begutachtungsleitlinien zur Kraftfahreignung, http://www. bast.de/DE/ Verkehrssicherheit/Fachthemen/BLL/BLL-hidden_node.html

- Directive of the European Union/Richtlinie der Europäischen Union, https://www.jurion.de/gesetze/eu/32 $016 / 1106$

The individual legal texts are presented in the long version of the guideline.

\section{Conflict of Interest}

The conflict of interest are listed in Table 11 of the S2e Diabetes and road traffic guideline (https:// www.awmf.org/uploads/tx_ szleitlinien/057-026m_S3_Diabetes-und-Straßenverkehr_2017-12. pdf).

German Diabetes Association Clinical Practice Guidelines

This is a translation of the DDG clinical practice guideline published in: Diabetologie 2019; 14 (Suppl 2): S278-S288, DOI https://doi. org/10.1055/a-0899-0207

\section{References}

[1] Bundesministerium der Justiz und für Verbraucherschutz Verordnung über die Zulassung von Personen zum Straßenverkehr (FahrerlaubnisVerordnung - FeV). 2014;

[2] Bundesanstalt für Straßenwesen (BASt) Begutachtungsleitlinien zur Kraftfahreignung. Bergisch Gladbach, Stand 24. Mai 2018;

[3] ArbSchG Arbeitsschutzgesetz Gesetz über die Durchführung von Maßnahmen des Arbeitsschutzes zur Verbesserung der Sicherheit und des Gesundheitsschutzes der Beschäftigten bei der Arbeit (Arbeitsschutz- gesetz- ArbSchG) vom 07.08.1996 (BGBl. I 1246), geändert durch Artikel 8 des Gesetzes vom 19.10.2013 (BGBI. I S. 3836). 2013; 